\title{
Modelling of the Electric Field Distribution in Deep Transcranial Magnetic Stimulation in the Adolescence, in the Adulthood, and in the Old Age
}

\author{
Serena Fiocchi, ${ }^{1}$ Michela Longhi, ${ }^{1}$ Paolo Ravazzani, ${ }^{1}$ Yiftach Roth, \\ Abraham Zangen, ${ }^{2}$ and Marta Parazzini ${ }^{1}$ \\ ${ }^{1}$ CNR Consiglio Nazionale delle Ricerche, Istituto di Elettronica e di Ingegneria dell'Informazione e delle Telecomunicazioni IEIIT, \\ Piazza Leonardo da Vinci 32, 20133 Milano, Italy \\ ${ }^{2}$ Department of Life Sciences, Ben-Gurion University of the Negev, 84105 Beersheba, Israel
}

Correspondence should be addressed to Marta Parazzini; marta.parazzini@ieiit.cnr.it

Received 19 October 2015; Revised 3 February 2016; Accepted 15 February 2016

Academic Editor: Dong Song

Copyright (c) 2016 Serena Fiocchi et al. This is an open access article distributed under the Creative Commons Attribution License, which permits unrestricted use, distribution, and reproduction in any medium, provided the original work is properly cited.

In the last few years, deep transcranial magnetic stimulation (dTMS) has been used for the treatment of depressive disorders, which affect a broad category of people, from adolescents to aging people. To facilitate its clinical application, particular shapes of coils, including the so-called Hesed coils, were designed. Given their increasing demand and the lack of studies which accurately characterize their use, this paper aims to provide a picture of the distribution of the induced electric field in four realistic human models of different ages and gender. In detail, the electric field distributions were calculated by using numerical techniques in the brain structures potentially involved in the progression of the disease and were quantified in terms of both amplitude levels and focusing power of the distribution. The results highlight how the chosen Hesed coil (H7 coil) is able to induce the maxima levels of E mainly in the prefrontal cortex, particularly for the younger model. Moreover, growing levels of induced electric fields with age were found by going in deep in the brain, as well as a major capability to penetrate in the deepest brain structures with an electric field higher than $50 \%, 70 \%$, and $90 \%$ of the peak found in the cortex.

\section{Introduction}

During the last decades, the prevalence of depression among a broad population [1] has led to increasing efforts to find new methods for alleviating the severe symptoms of that condition. Among them, transcranial magnetic stimulation (TMS) offers particularly appealing advantages, given mainly its noninvasive and painless use.

The efficacy of this technique is mediated by the electromagnetic induction of an electric field as described by Faraday's law. In brief, TMS is applied through short magnetic pulses delivered to the brain, produced by the passage of high electric current pulses in a coil placed over the patient's head [2].

The different shapes of the coil can modify the penetration depth and the focalization of the electric field and hence the likelihood of achieving specific targets in the brain.
The aetiology of depression disorders is still not completely clear, but it is unlikely to affect only a single brain region or a neurotransmitter system. More probably, the heterogeneous symptoms of depressive disorders are driven by different brain structures, which interact in complex neurocircuits [3]. Most of the researches on depression attribute the progression of mood disorders to the impairment of areas belonging to limbic neurocircuits [4]. They comprehend the prefrontal cortex (PFC), the cingulate cortex, the hippocampus, some subcortical structures implicated in the reward circuit (such as the amygdala, the nucleus accumbens, and the ventral tegmental area) [5], and their connections with diencephalic areas (the hypothalamus, the thalamus, and the midbrain), just to name a few.

In order to target specifically these structures and in the meantime to overcome the undesirable side effects led by the conventional TMS coils [6-8], lately, new coils have been 
designed, with the aim of effectively stimulating deep brain targets with the so-called deep TMS (dTMS). This is the case of the family of the Hesed (H-) coils [9-11]: their current paths have a complex three-dimensional structure, which minimizes the nontangential coils elements thus allowing stimulating more efficaciously the structures that are further away from the scalp.

A particular type of $\mathrm{H}$-coil, the $\mathrm{H} 7$ coil, recently used with positive outcomes in clinical trials $[12,13]$, is getting a prominent position among the other $\mathrm{H}$-coils. The $\mathrm{H} 7$ coil is based on a new design that pledges a direct stimulation of frontal brain regions (i.e., the PFC) and it is specifically designed to reach deep brain regions without increasing the electric field levels in the more superficial cortical regions.

In order to link the role of the neural circuits mentioned above in the progressions of the depressive disorder with the efficacy of dTMS treatment with $\mathrm{H}$-coils, a crucial role is played by the knowledge of the electric field distribution induced within the target tissues. In the meanwhile, the assessment of electric field (E) in different subjects could give peculiar information about a rationale and customized use of dTMS.

In this study we investigated, by a computational electromagnetic approach, the electric field induced by the $\mathrm{H} 7$ coil in the brain tissues involved in the depression disorders in four different human models of various ages and gender. With the specific aim of evaluating to which extent the age and the anatomical head morphology can affect the $\mathbf{E}$ distribution, we also evaluated its stimulation depth and focusing power over the four human models.

\section{Materials and Methods}

2.1. Human Models. Four high-resolution anatomical models of the virtual family [14] were used in this study: a child of 14 years of age (Louis), an adult female of 26 years of age (Ella), an adult male of 34 years of age (Duke), and an old male of 84 years of age (Glenn). Their different age allows considering and examining the potential application of dTMS by focusing on the main stages of body maturation and tissues diversity, that is, the adolescence, the adult age over both genders, and the old age. These human models have been developed by high-resolution segmentation of magnetic resonance (MR) images of healthy volunteers. They consist of up to 84 different tissues in the whole body and about 20 tissues at the brain level. Given the involvement in the pathogenesis of depression of some structures and subregions not distinguished within the human models used in this study, they were identified through a comparison with a brain atlas based on MR images. Specifically, in each human model, the left and the right amygdala, the ventral tegmental area (VTA), and the left and right nucleus accumbens have been included, all of them of particular interest for this study, together with the prefrontal cortex (PFC) and its subregions (i.e., the Dorsolateral Prefrontal Cortex, DLPFC, and the Medial Prefrontal Cortex, MPFC) and the anterior cingulate cortex. The dielectric properties of each tissue have been assigned according to literature data $[15,16]$ at the dTMS typical frequency.
2.2. Coil Configuration. In this study, we have modelled the H7 coil configuration. Briefly, it is formed by eight copper windings on each of the right and left wings, with a complete right-left symmetry in the coil configuration. In each wing, the eight windings are arranged on two layers of four windings each: the windings in the 2 nd layer are placed on top of the windings on the 1st layer and adjacent to them. In each layer, the four windings are concentric ellipses, whose major axis ranges from 140 (outer ellipse) to 75 (inner ellipse) $\mathrm{mm}$, and minor axis ranges from 125 to $70 \mathrm{~mm}$. The wire diameter, including isolation material, is of $5.1 \mathrm{~mm}$. The angle between the two planes on which the two groups of eight windings lie can be modified to conform to the shape and size of the head so that the coil windings are tangential to the head at the treatment position. The distance between the closest edges of adjacent couple of windings is $0.5 \mathrm{~cm}$; the minimum distance between the right and left wings is of $3 \mathrm{~cm}$.

Here the coil was placed in two different positions on specific anatomic sites of the head, according to the procedure commonly used in clinical practice. Firstly, it was placed over the leg motor cortex, to identify the minimum current able to generate in the cortical area of the motor cortex of tibialis anterior an electric field of $100 \mathrm{~V} / \mathrm{m}$. Then, the coil, fed with the same current, is moved $4 \mathrm{~cm}$ anteriorly and positioned over the frontal cortex (Figure 1).

The current delivered to the coil was a biphasic sinusoidal pulse at a frequency of $3 \mathrm{kHz}$.

2.3. Electric Field Numerical Simulations. The simulations were run using the simulation platform SEMCAD $\mathrm{X}$ (by SPEAG, http://www.speag.com/) [17], using the magneto quasi-static low frequency solver, which solves the BiotSavart law and is based on the scalar potential finite element (SPFE) method. In the low frequency range, the dimensions of the computational domain are smaller than the free space wavelength and we can apply the magneto quasi-static approximation. Hence, the magnetic vector potential $\mathbf{A}$ is decoupled from $\mathbf{E}$. Once $\mathbf{A}$ is calculated using the Biot-Savart law, electric field $\mathbf{E}$ can be determined by the scalar potential $\Phi$, which is given by

$$
-\nabla \cdot \sigma \nabla \Phi=j \omega \nabla \cdot(\sigma \mathbf{A})
$$

with $\sigma$ as the tissue conductivity and $\omega$ as the angular frequency of the field.

The computational domain was truncated just below the shoulders level of the human models, where the level of the electric fields induced by the coil can be considered negligible. A uniform hexahedral meshing algorithm, made available by the software [17], with a maximum step of $1 \mathrm{~mm}$, was chosen to allow the discretization of all tissues at the brain level, following an approach already used in dTMS literature [18]. It results in an applied mesh ranging from 57.5 to 117.3 million mesh cells.

2.4. Electric Field Characterization. The results will be presented in terms of both amplitude and spread of the electric field distribution. As to the amplitude, the maximum (named also "peak" in the following of this study) was taken as 


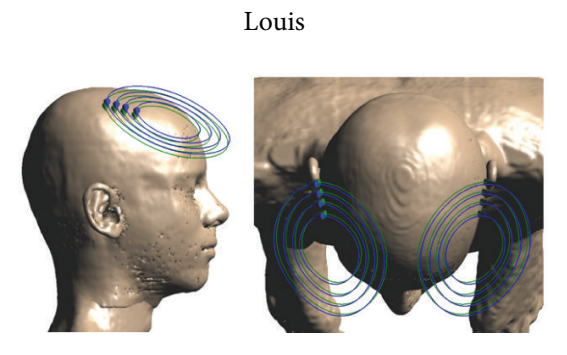

Duke
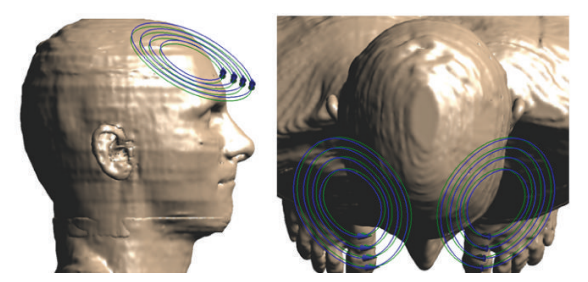

Ella
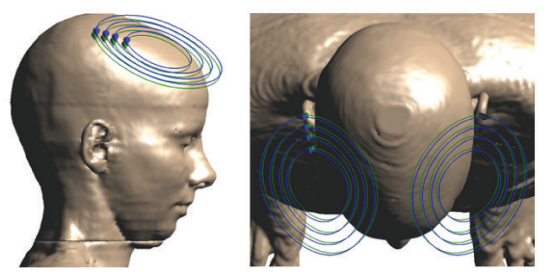

Glenn
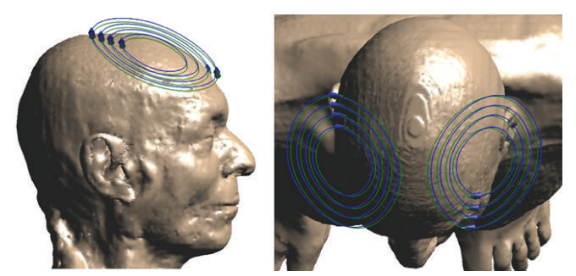

FIGURE 1: Sagittal and axial view of the four human models and relative position of the H7 coil. The green and blue ellipses indicate the four windings on the first and second layer for each wing.

a reference for the amplitude distribution analysis. The maximum corresponds to the 99th percentile of the distribution in the pertinent tissue to avoid any possible computational instabilities $[19,20]$.

The focusing power of the $\mathbf{E}$ amplitude distributions inside different brain regions has been also estimated and presented in terms of the percentage of volume of the tissue that is exposed to $\mathbf{E}$ amplitude equal to or greater than $50 \%$, $70 \%, 80 \%$, and $90 \%$ of its peak in the cortex (V50, V70, V80, and V90, resp.). These parameters give a quantitative estimation of the capability to concentrate high $\mathbf{E}$ in a small volume; therefore the smaller these volumes are, the more the field is focused.

In order to compute the capability of the $\mathrm{H} 7$ coil to penetrate in the brain with a given amplitude strength, we assessed the maximum depth from the cortical surface of the deepest point with $\mathbf{E}$ amplitude equal to or higher than $50 \%$ (d50), $70 \%$ (d70), and $90 \%$ (d90) of the peak of $\mathbf{E}$ in the cortex. In detail, a brain centre and the direction of penetration along the line passing through that centre and the identified deepest point were determined. Then the depth was calculated by means of the distance between the point and its projection over the cortical surface, along the direction of penetration, following a method proposed by Guadagnin and colleagues [18]. A schematic representation of this method and the location of $\mathrm{Cz}$, Nasion, and Inion of the 10-20 EEG system is shown in Figure 5 over Ella.

\section{Results}

Figure 2 shows $\mathbf{E}$ amplitude spatial distribution induced by the H7 coil in different brain tissues for all the human models. All colour maps are normalized to the peak of $\mathbf{E}$ amplitude distribution in the cortex for each model. From top to bottom, the normalized amplitude distributions are shown on the cortex (top row), the white matter (second row), the cerebellum, and the deep brain tissues such as the hippocampus, the hypothalamus, the midbrain, the thalamus, the pons, the amygdala, the nucleus accumbens, and the VTA (third row).

Although $\mathbf{E}$ amplitude distributions in the cortex, in the white matter, and in the deeper tissues among the human models show different trends that depend on the individual head morphology, the general trend of the spatial distributions of the field amplitude shares some gross characteristics among the different human models. In particular, their pattern is qualitatively comparable, with a widespread distribution toward the prefrontal lobes and a dramatic decrease (of about more of the $75 \%$ ) of the amplitude in the deepest tissues.

Figure 3 shows the descriptive statistic of $\mathbf{E}$ amplitude distributions in different brain tissues for all the four models. The intramodel description indicates a consistent contraction of the peak values passing from the cortex to the white matter, with a decrease of $48.8 \%, 23.4 \%, 41.9 \%$, and $32.2 \%$ for Louis, Ella, Duke, and Glenn, respectively, with respect to the cortex. These decreases are even more noticeable passing from the white matter to the deeper structures, where the percentages of reduction of the peak are $84.6 \%, 64.4 \%, 67.9 \%$, and $64.5 \%$ for Louis, Ella, Duke, and Glenn, respectively, with respect to the white matter. Similar behaviours are shown also by the median values with a decrease of them from the white matter to the deeper structures of $66.5 \%, 51.9 \%, 50.4 \%$, and $48.5 \%$ for Louis, Ella, Duke, and Glenn, respectively.

The location of the peaks varies across the various models: it occurs in the right DLPFC in Louis, in the left PFC in Ella, in the MPFC in Duke, and in the right PFC in Glenn. These data suggest a clear decreasing trend of the peak with the increasing of age, with a peak contraction ranging from about $40 \%$ for Duke and Ella to $47 \%$ for Glenn with respect to Louis. On the contrary, the comparison among the four panels of Figure 3 indicates an opposite trend in the deeper tissues, 

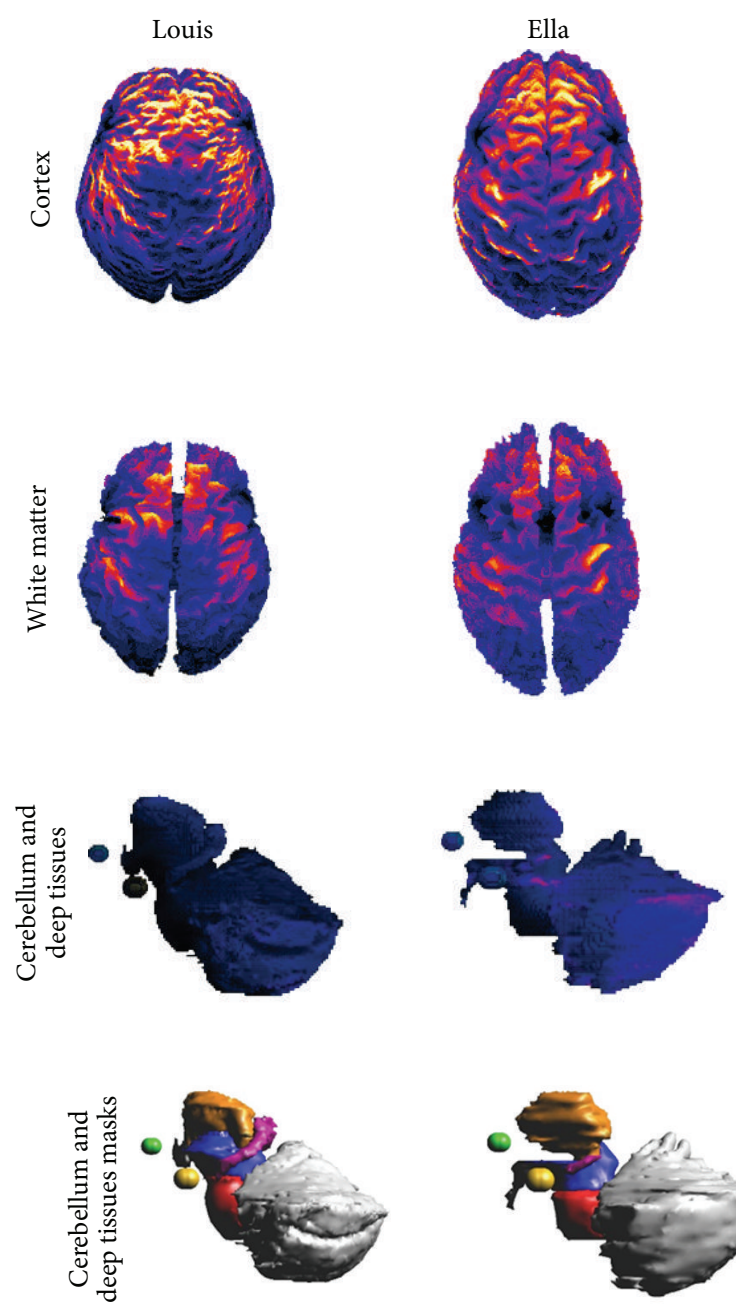

b)

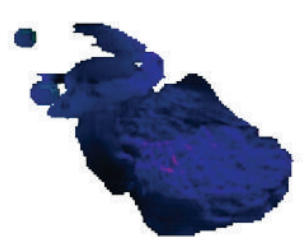

(c)

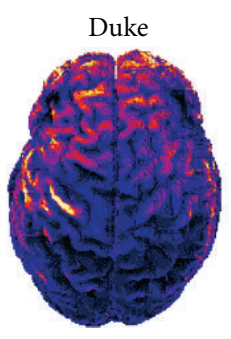

(a)
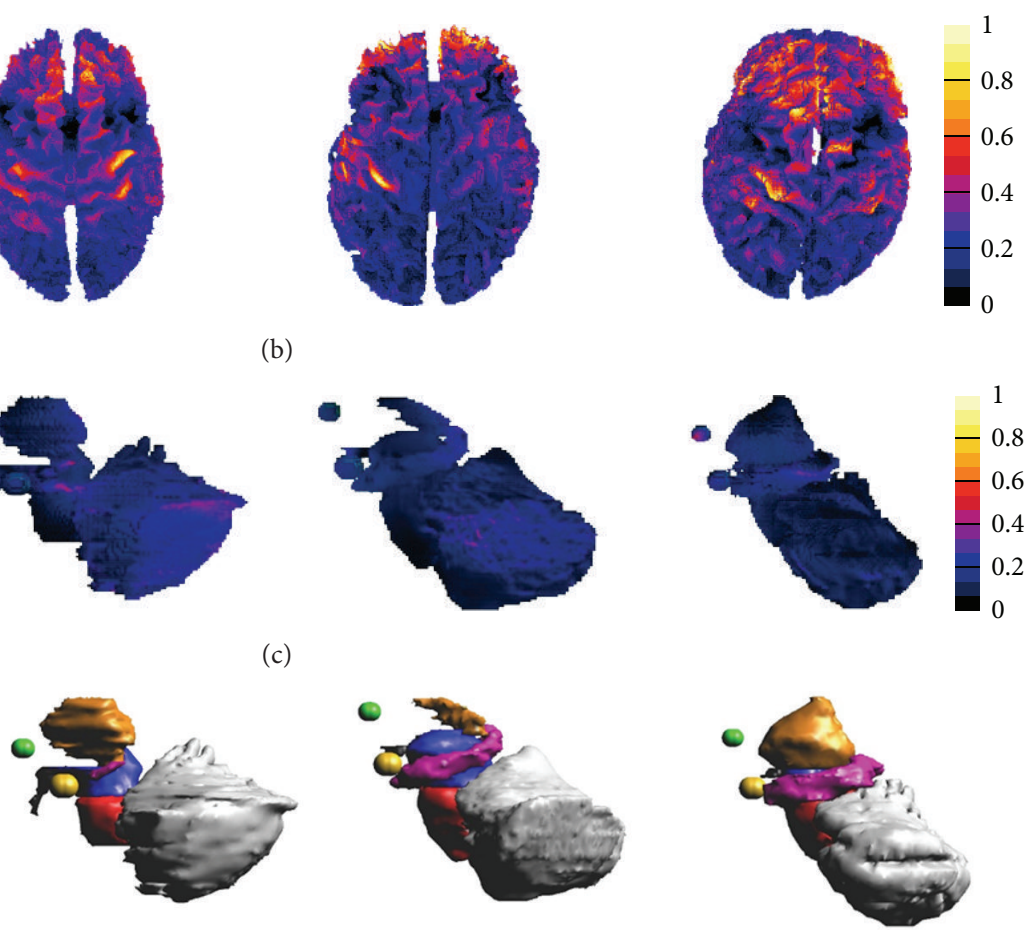

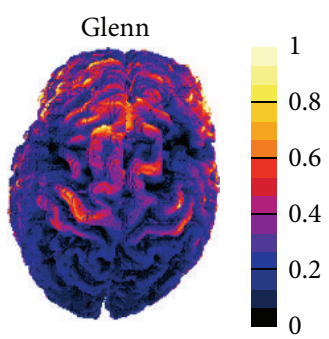

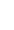

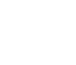

.

(d)

FIGURE 2: Surface amplitude distribution of $\mathbf{E}(\mathrm{V} / \mathrm{m})$ induced by the $\mathrm{H} 7$ coil in the cortex and white matter (1st and 2 nd rows) and in the cerebellum and deep brain tissues (3rd row) in Louis, Ella, Duke, and Glenn. For the sake of clarity, the 4th row shows the morphology of the deep brain tissues (the hippocampus in pink, the hypothalamus in black, the midbrain in blue, the thalamus in orange, the pons in red, the amygdala in yellow, and the nucleus accumbens in green) and the cerebellum (in grey). The amplitude field distributions of E are normalized with respect to the peak of $\mathbf{E}$ found in the cortex for each human model.

where the peak levels double passing from Louis to the adult models, in particular in the amygdala, the hippocampus, the hypothalamus, the nucleus accumbens, and the VTA.

To investigate better the characteristics of $\mathbf{E}$ amplitude distributions, Table 1 shows the V50 calculated over the cortex and its subregions and over the white matter. All the models show higher V50 in the DLPFC, with the exception of Duke for whom V50 is higher in the MPFC. Moreover, in the right DLPFC the V50 values are comparable in all the four models. A quite evident trend with age is shown in the left DLPFC, in which V50 ranges from $10.4 \%$ in Louis (the youngest model) to $20.1 \%$ in Glenn (the oldest model), and in the white matter, in which the V50 values almost quadruple from Louis to Glenn.

A more complete investigation of the focusing power is shown in Figure 4, as V70, V80, and V90 data estimated in the various brain regions of the prefrontal cortex taken into account in this study. The values found in the PFC and in the DLPFC are almost comparable among the models (with difference of $3 \%$ at maximum), whereas only for Duke the $\mathrm{H} 7$ coil is able to induce $\mathbf{E}$ amplitude higher than $90 \%, 80 \%$, and $70 \%$ of its peak in the cortex in volumes of the MPFC much higher than in the other models.

Figure 5 summarizes the penetration depth from the cortical surface of the deepest point with $\mathbf{E}$ amplitude equal to or higher than the $50 \%(\mathrm{~d} 50), 70 \%(\mathrm{~d} 70)$, and $90 \%(\mathrm{~d} 90)$ of the peak of $\mathbf{E}$ in the cortex, along a direction toward the brain centre, for all human models (in the figure a pictorial description of the brain centre identification is also shown).

The capability to penetrate in the cortex is the highest for Glenn, reaching almost $7 \mathrm{~cm}$ with an intensity higher than the $50 \%$ of the peak and almost $5 \mathrm{~cm}$ with the $90 \%$ of the peak, whereas the lowest one is found for Louis, with a decrease with respect to Glenn's penetration depth of 3.5, 5.1, 
TABLE 1: Percentage of the volume of different brain tissue where the amplitude of $\mathbf{E}$ is greater than 50\% (V50) of the peak of $\mathbf{E}$ in the cortex, in each human model.

\begin{tabular}{|c|c|c|c|c|c|c|c|}
\hline V50 (\%) & Cortex & White matter & Left PFC & Right PFC & Left DLPFC & Right DLPFC & MPFC \\
\hline Louis & 12.9 & 1.0 & 7.1 & 8.3 & 10.4 & 12.8 & 0.7 \\
\hline Ella & 5.3 & 1.7 & 10.7 & 11.3 & 12.9 & 11.9 & 5.9 \\
\hline Duke & 4.7 & 2.7 & 17.9 & 17.9 & 12.2 & 11.1 & 24.5 \\
\hline Glenn & 3.6 & 4.4 & 8.7 & 10.7 & 20.1 & 11.4 & 10.2 \\
\hline
\end{tabular}
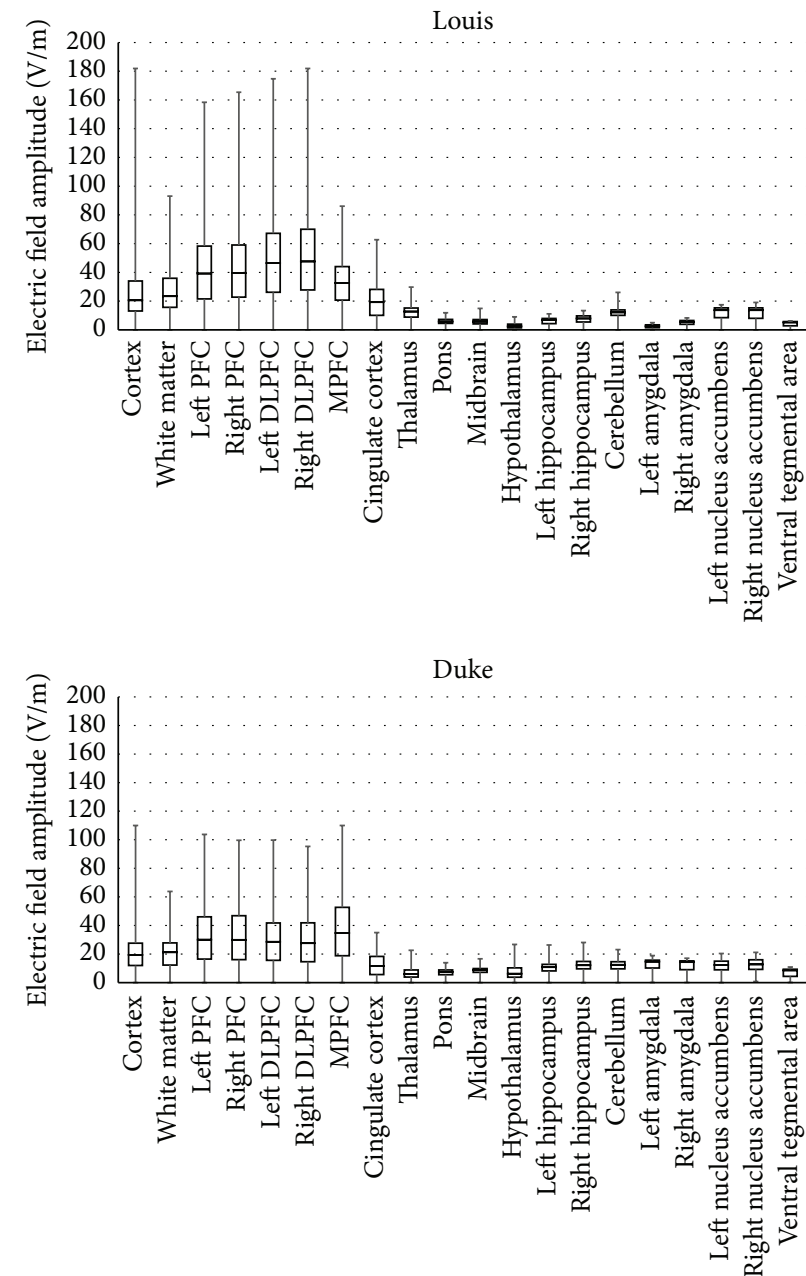
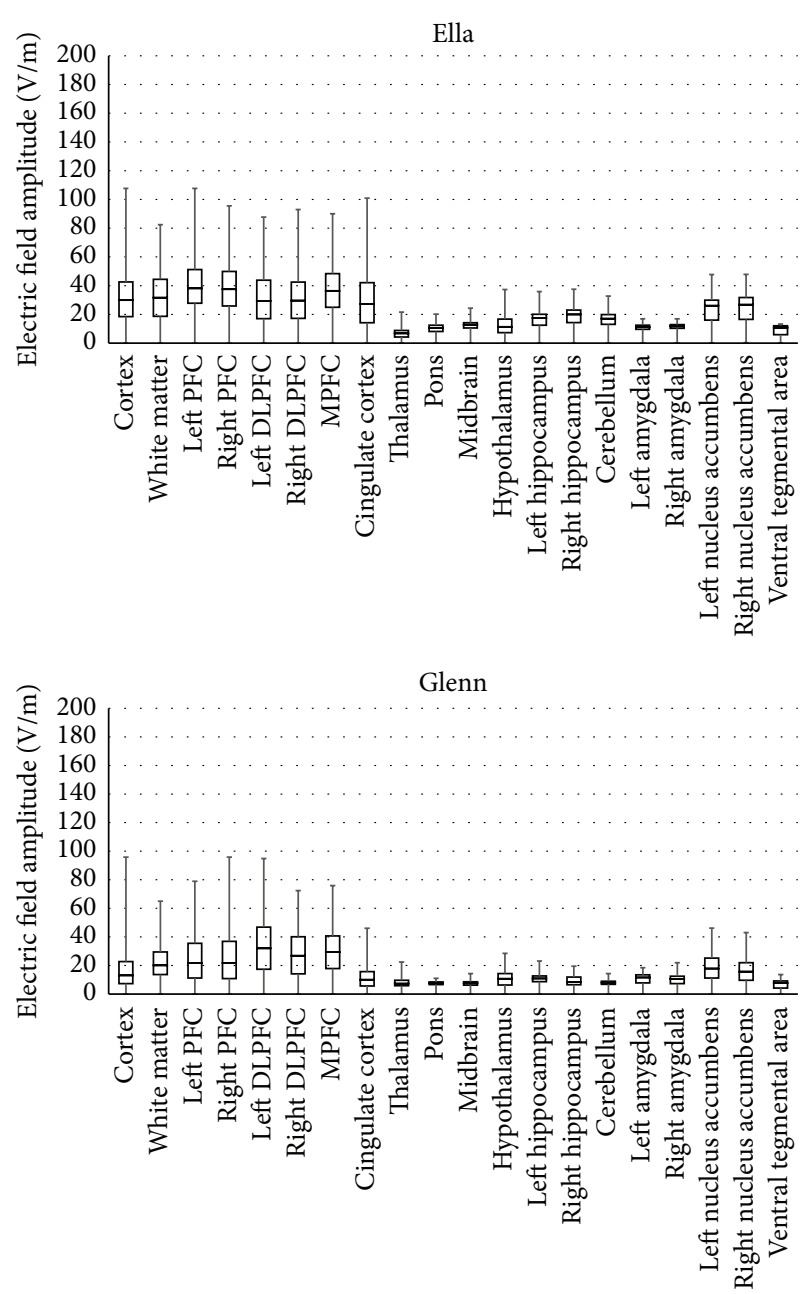

FIgURE 3: Descriptive statistic of $\mathbf{E}$ amplitude distributions $(\mathrm{V} / \mathrm{m})$ in different brain regions for all the four models.

and 4.9 times for $\mathrm{d} 50, \mathrm{~d} 70$, and $\mathrm{d} 90$, respectively. Moreover, it is higher in Ella than in Duke (with an average increase of $1 \mathrm{~cm}$ among the three distances), even if both models present a very slow decay (lower than the $10 \%$ passing from $\mathrm{d} 50$ to d90) among all the three depths of $\mathbf{E}$.

\section{Discussion and Conclusions}

The consistent adoption of dTMS to treat symptoms of depressive disorders and the positive results attained by the clinical applications of that technique [21-27] have led to the introduction of new stimulating devices, specifically designed for inducing sufficient level of $\mathbf{E}$ in the deep brain structures involved in the progression of the disease. Among them, the $\mathrm{H} 7$ coil is a new-concept device, recently introduced in the clinical practice $[12,13]$. This study aims at first assessing $\mathbf{E}$ amplitude distribution induced in these brain targets by that specific dTMS coil for the treatment of depressive disorder. Moreover, the effect of the morphological variability, even linked to age, has been also addressed. In view of the influence of the wide anatomical variability among subjects that could suffer from depressive symptoms, $\mathbf{E}$ distribution induced by the H7 coil has been compared indeed among subjects of different ages (from 14 to 84 years) and gender.

The results of this study are as such to indicate that the maxima levels of $\mathbf{E}$, as expected, occur mainly in the prefrontal cortex, identified as the main cortical region critically involved in the depressive disorders and in other brain 


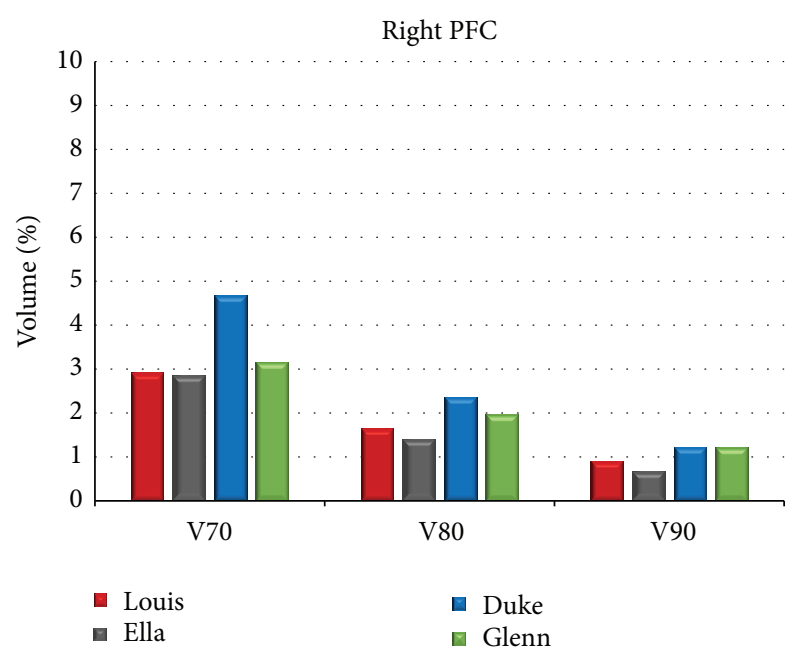

(a)

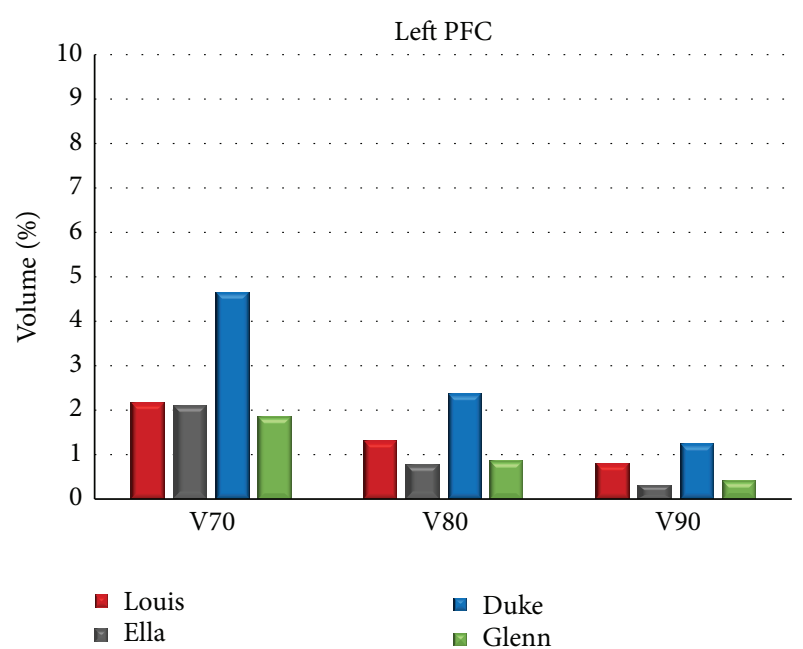

(b)

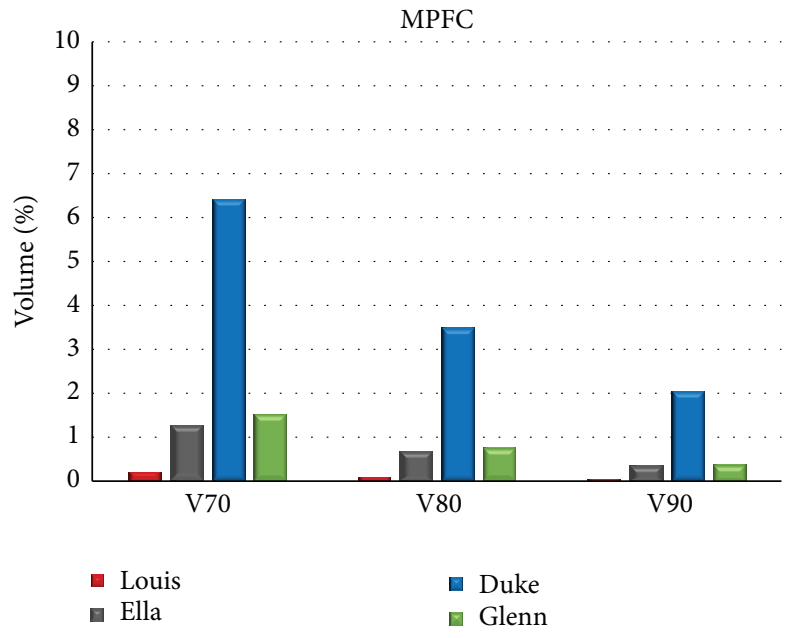

(c)

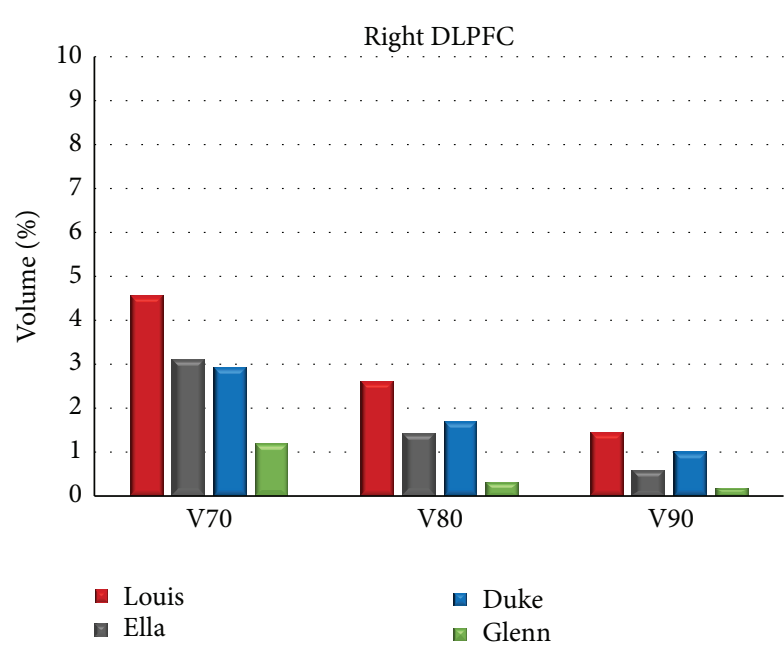

(d)

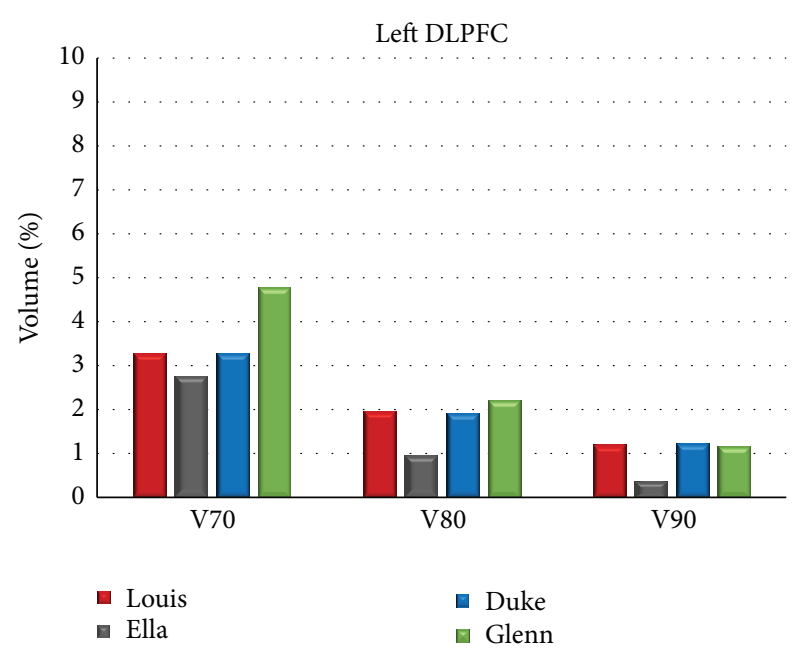

(e)

FIGURe 4: Percentage of the volume of the PFC (top panels), of the Medial Prefrontal Cortex (MPFC, panel in the middle), and of the Dorsolateral Prefrontal Cortex (DLPFC, bottom panels), showing an E amplitude higher than 70\% (V70), 80\% (V80), and 90\% (V90) of the $\mathbf{E}$ peak in the cortex. 


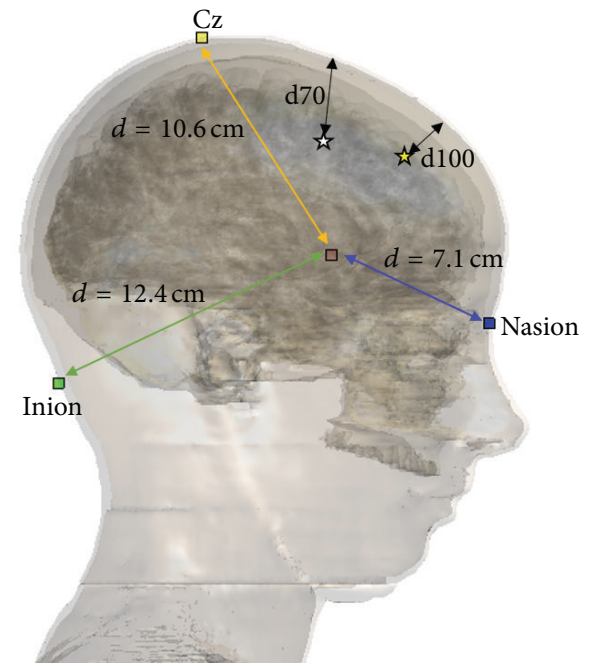

(a)

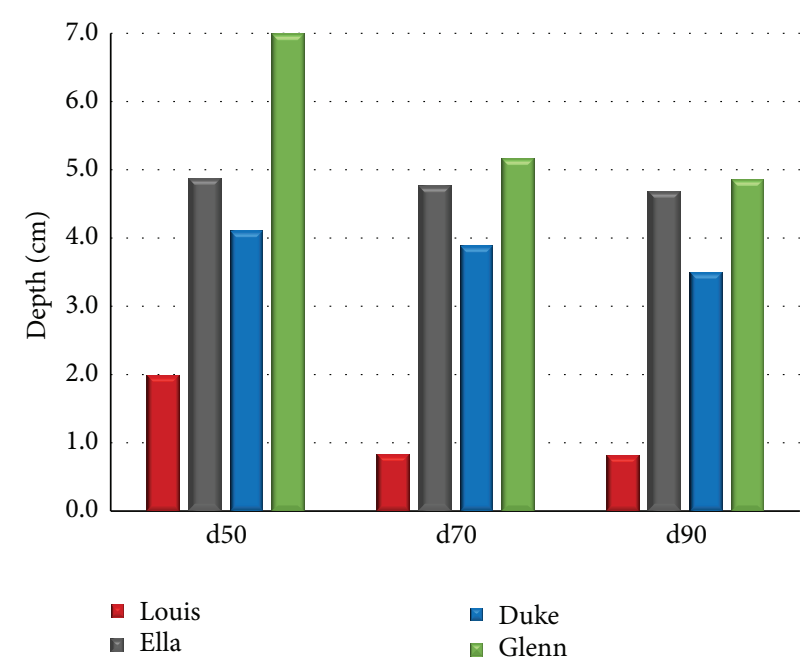

(b)

FIGURE 5: (a) Pictorial representation of the penetration depth calculation method, including the distance between the brain centre and the $\mathrm{Cz}$, the Inion, and the Nasion of the 10-20 EEG system reference points for the Ella model (taken as an example). (b) Depth (cm) of the deepest point at $50 \%$, at $70 \%$, and at $100 \%$ of the peak of $\mathbf{E}$ from the cortical surface of the four models.

disorders such as obsessive-compulsive disorder (OCD), and close to the area over which the coils are placed (see Figures 2 and 3). E amplitude distributions show a high degree of variability among the subjects, with an evident influence of the dimension of the subject head, which helps to achieve the highest $\mathbf{E}$ amplitudes in the Louis head model, that is, the smallest one. $\mathbf{E}$ is also clearly influenced by the age of the modelled subjects: the lowest $\mathbf{E}$ levels have been found in Glenn, the oldest head model. This could be due to the cortical atrophy that affects elderly people [28] and should be strongly taken into account when dTMS is applied to the clinical treatment of neural diseases such as dementias.

The same behaviour can be found in the white matter (Figure 3), with the highest $\mathbf{E}$ peaks for Louis and the lowest for Duke and Glenn. Moreover, both the median and peak $\mathrm{E}$ levels in the cingulate cortex, belonging to the limbic lobe and strictly associated with the development of the obsessivecompulsive disorder, are highest in the female model (Ella), decrease in Louis, and are close to the levels of the deepest structure in the male and elderly models (Duke and Glenn).

These findings could be of overarching importance, since most of the connections of the neural circuits involved in the depression and in the OCD occur through the myelinated nerve cells projections of the white matter and through the cingulum.

In the deeper tissues, that is, in particular in the ones that are considered central for maintaining emotional stability and whose malfunction is linked to the pathophysiology of depression (i.e., the amygdala, the hippocampus, the hypothalamus, the nucleus accumbens, and the VTA), E is lower in the youngest model and shows comparable values in the adult models whereas it shows the highest value in Glenn. That implies a major decrease of $\mathbf{E}$ amplitude with the distance for the youngest model. These considerations are fully in line with the investigation of the penetration depth shown in Figure 5, in which Louis shows the lowest value of penetration depth and Glenn shows the highest one. That decrease could be due to the major thickness of the young prefrontal cortex (even 35\% larger than that for adults and $50 \%$ with respect to elderly). On the contrary, the prefrontal atrophy, which characterizes the cortex in the elderly with the consequent increase of the CSF [29], could justify the high levels of $\mathbf{E}$ in the deepest tissues in Glenn.

The tissue localization of $\mathbf{E}$ peaks is different across the models (see Figure 3), whereas the focusing power (Figure 4) suggests a slight prevalence of the right side. This behaviour could be in line with some considerations about the therapeutic effects of dTMS, discussed in some studies [21, 30], which found laterality effect with a gain from excitatory dTMS over the left DLPFC and inhibitory dTMS over the right DLPFC. In any case, the $\mathrm{H} 7$ coil shows good capability to focus high levels of electric field in a very circumscribed volume, the focusing power being very high in all PFC areas (Figure 4), in particular in the youngest model. Lastly, the slighter major capability to penetrate into the deepest regions of Ella compared to Duke (Figure 5) could indicate some gender difference that can favour the application of these techniques in females, even though this should be better investigated in the future.

The variation of the levels and of the spread of the electric field levels across the models could have some important implications on the degree of neuromodulation within the brain tissues and, more specifically, on the excitability of the pyramidal cells around the target area. Indeed, since the size and the direction of the targeted cells and their axons are likely to vary across the model, this could affect the extent of the stimulation region [31].

However, since during their practices for brain targeting the clinicians are more interested in a general ranking of the brain regions where the field levels are higher instead of the minute details of current flow patterns, it could be sufficient to use some "reference human models" such as 
"adult" or "adolescent" and "elderly" in the planning of a dTMS treatment [32]. Our approach, therefore, could be an alternative to the use of individualized or customized models, to tune finely the dTMS treatment. Although this latter approach is in principle the optimal one, building a specific model for each patient can result in an extremely time-consuming and very expensive procedure.

In conclusion, this study shows that $\mathrm{H} 7$ coil is able to induce levels of electric field in the typical brain target areas for the treatment of depressive disorders comparable with the ones found for other $\mathrm{H}$-family coils [33]. This conclusion is still valid also considering the effect of age. Indeed, the results presented here for Glenn and Duke are fully in line with the outcomes found for the same models by the $\mathrm{H} 1$ coil, that is, another coil of the H-family [33], which showed a slight decrease of the electric field levels in the prefrontal cortex and higher capability to reach deeper target in the brain for the elderly.

\section{Competing Interests}

Professor Zangen and Dr. Roth are inventors of the deep TMS technology and have financial interests in Brainsway, which produces the deep TMS H-coil systems. All the other authors have no potential conflict of interests to be disclosed.

\section{Acknowledgments}

The authors wish to thank Schmid \& Partner Engineering AG (http://www.speag.com/) for having provided the simulation software SEMCAD X.

\section{References}

[1] C. J. L. Murray and A. D. Lopez, "Alternative projections of mortality and disability by cause 1990-2020: global burden of disease study," The Lancet, vol. 349, no. 9064, pp. 1498-1504, 1997.

[2] Y. Roth, F. Padberg, and A. Zangen, "Transcranial magnetic stimulation of deep brain regions: principles and methods," Advances in Biological Psychiatry, vol. 23, pp. 204-224, 2007.

[3] E. J. Nestler, M. Barrot, R. J. DiLeone, A. J. Eisch, S. J. Gold, and L. M. Monteggia, "Neurobiology of depression," Neuron, vol. 34, no. 1, pp. 13-25, 2002.

[4] M. M. Rive, G. van Rooijen, D. J. Veltman, M. L. Phillips, A. H. Schene, and H. G. Ruhé, "Neural correlates of dysfunctional emotion regulation in major depressive disorder. A systematic review of neuroimaging studies," Neuroscience and Biobehavioral Reviews, vol. 37, no. 10, pp. 2529-2553, 2013.

[5] E. Palazidou, “The neurobiology of depression," British Medical Bulletin, vol. 101, no. 1, pp. 127-145, 2012.

[6] M. Nadeem, T. Thorlin, O. P. Gandhi, and M. Persson, "Computation of electric and magnetic stimulation in human head using the 3-D impedance method," IEEE Transactions on Bio-Medical Engineering, vol. 50, no. 7, pp. 900-907, 2003.

[7] L. Maizey, C. P. G. Allen, M. Dervinis et al., "Comparative incidence rates of mild adverse effects to transcranial magnetic stimulation," Clinical Neurophysiology, vol. 124, no. 3, pp. 536$544,2013$.
[8] J. Leitão, A. Thielscher, S. Werner, R. Pohmann, and U. Noppeney, "Effects of parietal TMS on visual and auditory processing at the primary cortical level-a concurrent TMSfMRI study," Cerebral Cortex, vol. 23, no. 4, pp. 873-884, 2013.

[9] Y. Roth, A. Zangen, and M. Hallett, "A coil design for transcranial magnetic stimulation of deep brain regions," Journal of Clinical Neurophysiology, vol. 19, no. 4, pp. 361-370, 2002.

[10] A. Zangen, Y. Roth, B. Voller, and M. Hallett, "Transcranial magnetic stimulation of deep brain regions: evidence for efficacy of the H-coil," Clinical Neurophysiology, vol. 116, no. 4, pp. 775-779, 2005.

[11] Y. Levkovitz, Y. Roth, E. V. Harel, Y. Braw, A. Sheer, and A. Zangen, "A randomized controlled feasibility and safety study of deep transcranial magnetic stimulation," Clinical Neurophysiology, vol. 118, no. 12, pp. 2730-2744, 2007.

[12] G. Kranz, E. A. Shamim, P. T. Lin, G. S. Kranz, and M. Hallett, "Transcranial magnetic brain stimulation modulates blepharospasm: a randomized controlled study," Neurology, vol. 75, no. 16, pp. 1465-1471, 2010.

[13] D. Dalla Libera, B. Colombo, E. Coppi et al., "Effects of highfrequency repetitive transcranial magnetic stimulation (rTMS) applied with $\mathrm{H}$-coil for chronic migraine prophylaxis," Clinical Neurophysiology, vol. 122, pp. S145-S146, 2011.

[14] L. Carmi, U. Alyagon, R. Dar, J. Zohar, and A. Zangen, "Deep transcranial magnetic stimulation (Tms) in obsessive compulsive disorder (Ocd) patients," European Psychiatry, vol. 30, supplement 1, p. 794, 2015.

[15] S. Gabriel, R. W. Lau, and C. Gabriel, "The dielectric properties of biological tissues. II. Measurements in the frequency range $10 \mathrm{~Hz}$ to $20 \mathrm{GHz}$, Physics in Medicine and Biology, vol. 41, no. 11, pp. 2251-2269, 1996.

[16] C. Gabriel, A. Peyman, and E. H. Grant, "Electrical conductivity of tissue at frequencies below $1 \mathrm{MHz}$," Physics in Medicine and Biology, vol. 54, no. 16, pp. 4863-4878, 2009.

[17] SEMCAD X v14.8 by SPEAG, Schmid \& Partner Engineering AG, Zürich, Switzerland, http://www.speag.com/.

[18] V. Guadagnin, M. Parazzini, S. Fiocchi, I. Liorni, and P. Ravazzani, "Deep transcranial magnetic stimulation: modeling of different coil configurations," IEEE Transactions on Biomedical Engineering, 8 pages, 2015.

[19] M. Parazzini, S. Fiocchi, I. Liorni et al., "Modeling the current density generated by transcutaneous spinal direct current stimulation (tsDCS)," Clinical Neurophysiology, vol. 125, no. 11, pp. 2260-2270, 2014.

[20] M. Parazzini, S. Fiocchi, I. Liorni, A. Priori, and P. Ravazzani, "Computational modeling of transcranial direct current stimulation in the child brain: implications for the treatment of refractory childhood focal epilepsy," International Journal of Neural Systems, vol. 24, no. 2, Article ID 1430006, 10 pages, 2014.

[21] Y. Levkovitz, E. V. Harel, Y. Roth et al., "Deep transcranial magnetic stimulation over the prefrontal cortex: evaluation of antidepressant and cognitive effects in depressive patients," Brain Stimulation, vol. 2, no. 4, pp. 188-200, 2009.

[22] Y. Levkovitz, A. Sheer, E. V. Harel et al., "Differential effects of deep TMS of the prefrontal cortex on apathy and depression," Brain Stimulation, vol. 4, no. 4, pp. 266-274, 2011.

[23] M. Isserles, O. Rosenberg, P. Dannon et al., "Cognitiveemotional reactivation during deep transcranial magnetic stimulation over the prefrontal cortex of depressive patients affects antidepressant outcome," Journal of Affective Disorders, vol. 128, no. 3, pp. 235-242, 2011. 
[24] O. Rosenberg, N. Shoenfeld, A. Zangen, M. Kotler, and P. N. Dannon, "Deep TMS in a resistant major depressive disorder: a brief report," Depression and Anxiety, vol. 27, no. 5, pp. 465-469, 2010.

[25] O. Rosenberg, Y. Roth, M. Kotler, A. Zangen, and P. Dannon, "Deep transcranial magnetic stimulation for the treatment of auditory hallucinations: a preliminary open-label study," Annals of General Psychiatry, vol. 10, article 3, 2011.

[26] E. V. Harel, L. Rabany, L. Deutsch, Y. Bloch, A. Zangen, and Y. Levkovitz, "H-coil repetitive transcranial magnetic stimulation for treatment resistant major depressive disorder: an 18-week continuation safety and feasibility study," World Journal of Biological Psychiatry, vol. 15, no. 4, pp. 298-306, 2014.

[27] E. V. Harel, A. Zangen, Y. Roth, I. Reti, Y. Braw, and Y. Levkovitz, " $\mathrm{H}$-coil repetitive transcranial magnetic stimulation for the treatment of bipolar depression: an add-on, safety and feasibility study," World Journal of Biological Psychiatry, vol. 12, no. 2, pp. 119-126, 2011.

[28] Z. Nahas, X. Li, F. A. Kozel et al., "Safety and benefits of distance-adjusted prefrontal transcranial magnetic stimulation in depressed patients 55-75 years of age: a pilot study," Depression and Anxiety, vol. 19, no. 4, pp. 249-256, 2004.

[29] S. K. Kessler, P. Minhas, A. J. Woods, A. Rosen, C. Gorman, and M. Bikson, "Dosage considerations for transcranial direct current stimulation in children: a computational modeling study," PLoS ONE, vol. 8, no. 9, Article ID e76112, 2013.

[30] A. A. Gershon, P. N. Dannon, and L. Grunhaus, "Transcranial magnetic stimulation in the treatment of depression," The American Journal of Psychiatry, vol. 160, no. 5, pp. 835-845, 2003.

[31] B. D. Goodwin and C. R. Butson, "Subject-specific multiscale modeling to investigate effects of transcranial magnetic stimulation," Neuromodulation: Technology at the Neural Interface, vol. 18, no. 8, pp. 694-704, 2015.

[32] S. S. Shahid, M. Bikson, H. Salman, P. Wen, and T. Ahfock, "The value and cost of complexity in predictive modelling: role of tissue anisotropic conductivity and fibre tracts in neuromodulation," Journal of Neural Engineering, vol. 11, no. 3, Article ID 036002, 2014.

[33] S. Fiocchi, M. Parazzini, I. Liorni, Y. Roth, A. Zangen, and P. Ravazzani, "Deep transcranial magnetic stimulation for the treatment of neuropsychiatric disorders in elderly people: electric field assessment," in Proceedings of the International Conference on Electromagnetics in Advanced Applications (ICEAA '15), pp. 448-451, IEEE, Torino, Italy, September 2015. 


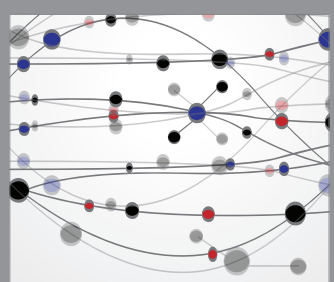

The Scientific World Journal
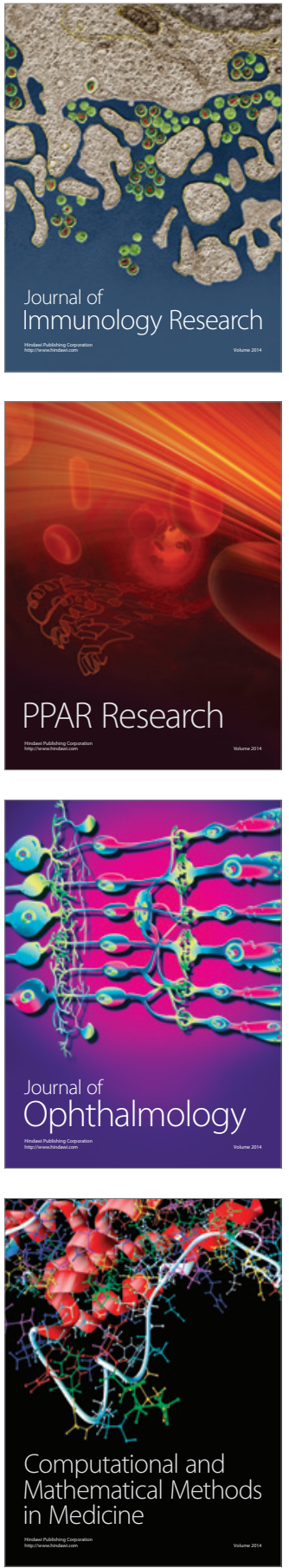

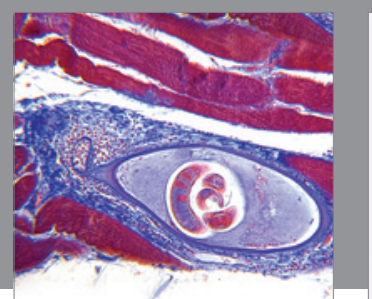

Gastroenterology Research and Practice

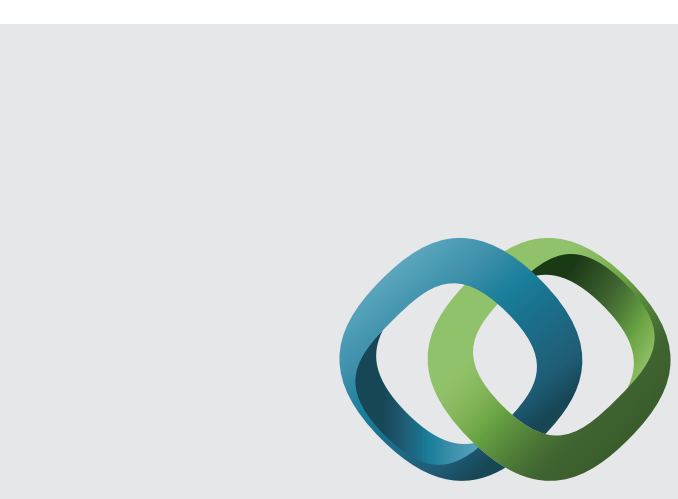

\section{Hindawi}

Submit your manuscripts at

http://www.hindawi.com
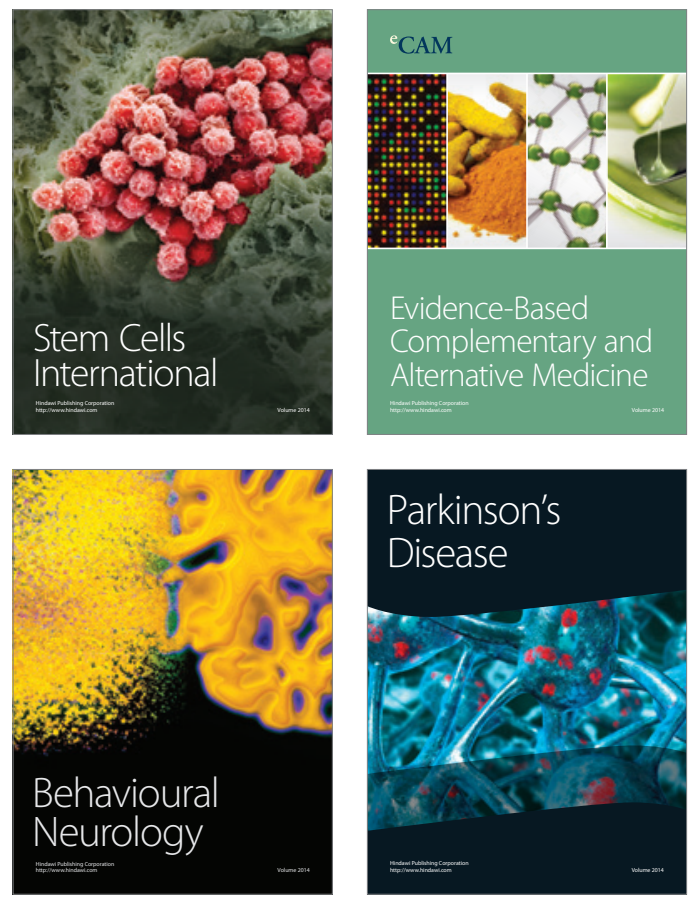
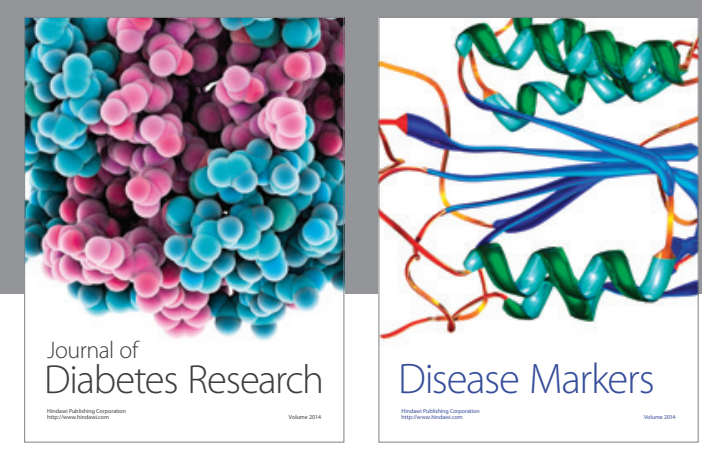

Disease Markers
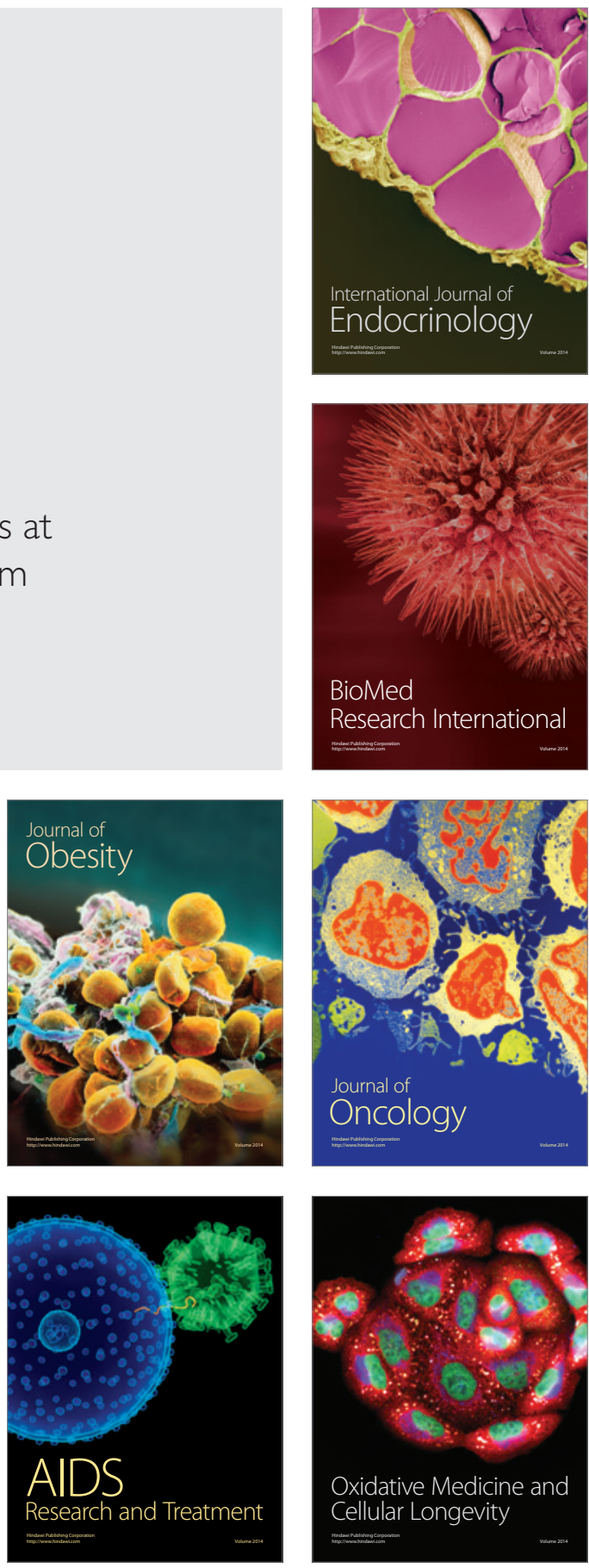